\title{
Quantitative Determination of Luminescent Coupling in Multijunction Solar Cells from Spectral Photovoltage Measurements
}

\author{
D. Fuertes Marrón, ${ }^{*}$ E. Barrigón, ${ }^{\dagger}$ M. Ochoa, and I. Artacho \\ Instituto de Energía Solar, Universidad Politécnica de Madrid, ETSI Telecomunicación, \\ Ciudad Universitaria s/n, 28040 Madrid, Spain \\ (Received 8 April 2016; revised manuscript received 15 April 2016)
}

\begin{abstract}
We present a simple method to quantify the magnitude of luminescent coupling (LC) between stacked subcells in multijunction photovoltaic devices. The effect of luminescence produced at high-band-gap subcells on underlying low-gap units within the same device can be directly accessed as a measurable opencircuit voltage difference by comparing two photovoltage spectra. Additionally, our study unambiguously identifies LC as the modulation mechanism across multijunction solar cells generating a response from buried subcells in photoreflectance measurements.
\end{abstract}

DOI:

\section{INTRODUCTION}

Multijunction solar cells (MJSCs) based on III-V semiconductors are currently the most efficient devices for photovoltaic energy conversion [1]. Despite its technological complexity, monolithic integration is the standard procedure in the design of these devices, by which subcells based on semiconductors of different energy band gaps are electrically connected in series with tunnel junctions, by piling up a 3 multilayer structure. The resulting two-terminal devices are convenient for subsequent electrical connection and module processing but complicate the characterization and quality assessment of specific parts or individual junctions within the device. Nonetheless, current-voltage $(J-V)$ characteristics, quantum efficiency (QE), and electro- or photoluminescence are routinely used in MJSC characterization, providing useful information of the optoelectronic properties of the subcells [2,3]. Monolithic integration imposes, in addition, characteristic constraints on the operation of devices, particularly concerning current matching between subcells under a given spectrum, in order to optimize its performance. Such constraints, affecting a large number of layers in the structure, translate into a tight set of growth and material parameters. Ideally, the grower would count with a bunch of characterization techniques that would permit independent assessment of each part of the device; however, such parameters are very often interrelated, affecting other parts in the device and complicating any separated diagnostics attempted.

One subtle difficulty in the optimization and characterization of subcells for current matching is luminescent coupling (LC): Band-to-band radiative recombination events at top cells generate light that can be reabsorbed

\footnotetext{
*dfuertes@ies-def.upm.es

"Present address: Division of Solid State Physics-Nano Lund, Lund University, SE-22100 Lund, Sweden.
}

at underlying lower-gap subcells and effectively contribute to the photocurrent. This additional photocurrent must be thus accounted for when designing high-efficiency devices, as to ensure operation as close as possible to current matching. As LC depends on the actual operation point of top cells, no single solution in the form of a closed set of parameters is expected as to ensure optimal performance under arbitrary illumination conditions. Still, the availability of a simple and reliable diagnostics tool capable of quantifying the magnitude of LC and the associated increase of photocurrent resulting from it is of high value. The implications of LC in the characterization, operation, and optimization of MJSCs are currently a matter of active research [4-8]. In what follows, we propose a simple method of quantifying LC as a measurable photovoltage generated at low-gap subcells. This approach has two distinct advantages: It (i) can easily be implemented in current setups for standard MJSC characterization and (ii) provides direct access to the magnitude of quasiFermi level splitting of subcells operating under LC effects, from which the optoelectronic response can be predicted.

\section{THEORETICAL BACKGROUND}

The proposed method is based on the measurement of the photovoltage generated in the device under two different pump-and-probe illumination sources. From Shockley's diode equation, the open-circuit voltage can be expressed as a function of the photogenerated currents. For multijunction devices, the overall open-circuit voltage expression, considering a single-diode model for each subcell, will include the summation of as many diode terms as junctions forming the device. Each diode term consists of a log factor, containing the currents, multiplied by a prefactor, the product of the corresponding diode ideality factor $n$ and the thermal voltage $k T / q$. For example, the photovoltage of a two-junction solar cell can be expressed as 


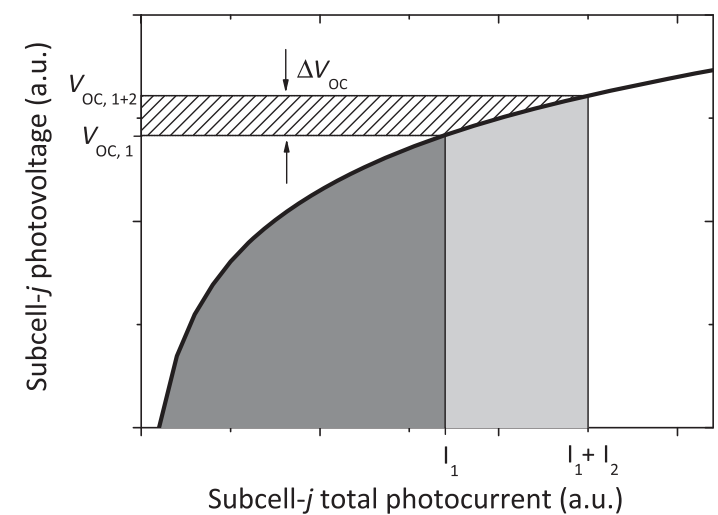

F1:1 FIG. 1. Logarithmic dependency of photovoltage upon photoF1:2 generated current in the $j$ subcell under two illumination sources F1:3 generating currents $I_{1}$ and $I_{2}$, respectively. The increase in overall F1:4 photovoltage results from current summation inside the log term.

$$
\begin{aligned}
V_{\mathrm{OC}}= & \frac{n^{\mathrm{TC}} k T}{q} \ln \left(\frac{\sum_{\alpha} I_{\alpha}^{\mathrm{TC}}(\lambda)}{I_{0}^{\mathrm{TC}}}+1\right) \\
& +\frac{n^{\mathrm{BC}} k T}{q} \ln \left(\frac{\sum_{\beta} I_{\beta}^{\mathrm{BC}}(\lambda)}{I_{0}^{\mathrm{BC}}}+1\right),
\end{aligned}
$$

where the first and second term on the right correspond to the top (TC) and bottom cell (BC), respectively, and $\alpha$ and $\beta$ indicate different current contributions at each diode junction stemming from different light sources. In what follows, we consider the case $I_{\alpha, \beta} \gg I_{0}$ and neglect the +1 terms in the logarithms.

The effect of more than a single illumination source inducing photogeneration on the photovoltage of a given subcell is illustrated in Fig. 1. The curve represents the logarithmic dependence of the output voltage on the photocurrent $I_{1}$. Adding a second light source contributing $I_{2}$ increases $V_{\mathrm{OC}}$ to a magnitude proportional to the logarithm of the sum of the currents $I_{1}+I_{2}$. The magnitude $\Delta V_{\mathrm{OC}}$ thus corresponds to the additional contribution generated by $I_{2}$. It will be shown that under certain illumination conditions such an increase in the recorded open-circuit voltage can be unambiguously ascribed to the effect of luminescent coupling between subcells in multijunction devices, thereby providing a simple way to estimate its magnitude.

\section{EXPERIMENT}

Simultaneous photovoltage and photoreflectance (PR) measurements are performed on a $(\mathrm{Ga}, \mathrm{In}) \mathrm{P} / \mathrm{GaAs}$ tandem solar cell grown by MOVPE. The application of spectral photovoltage measurements to single-junction solar cells and its relationship to the spectral response of the shortcircuit current is discussed by Mackel and Cuevas [9], although our scope and method are different in this work. Additionally, PR is used in this work as a complementary technique, supporting our main conclusions drawn from photovoltage measurements, as explained later on. PR belongs to the family of modulation spectroscopies [10], which consist of the measurement of small changes in the optical properties of the samples under study driven by applied perturbations, like electric fields, strain, or temperature gradients. In PR, such perturbations are induced by means of carrier photogeneration by a pump beam of sufficient photon energy. The photogenerated excess carriers diffuse and screen electric fields already present at space-charge regions within the semiconductor, inducing small changes in the dielectric constant of the material that are in turn recorded by means of a probe light beam as slight changes in the reflectance of the specimen.

The sample structure under study is shown schematically in Table I, and further details on the growth method can be found in Ref. [11]. The sample is provided with a Ni pad on the front side to permit electrical contact, and In is used to fix the sample back surface onto a Cu-disk holder. The estimated efficiency of the device is $25 \%$ under one-sun illumination, based on $\mathrm{QE}$ results and accounting for the lack of a front metal grid and antireflection coating (in order to facilitate PR measurements), as well as a larger effective area (approximately $0.25 \mathrm{~cm}^{2}$ ) as compared to $1-\mathrm{mm}^{2}$ standard devices. Metal tips on the front and rear contacts are used connected to a voltmeter to record the spectral photovoltage during the measurements. Sharma and Hosea [12] report on photovoltage results obtained using contact and contactless measurements, and we use a similar setup in this work. The photovoltage $V_{\mathrm{OC}}$ reading is fed to an analogto-digital converter and incorporated to the data-acquisition control during PR scans. Measurements are carried out illuminating the front side of the sample simultaneously with two light sources: the $325-\mathrm{nm}$ line of a $15-\mathrm{mW} \mathrm{HeCd}$ laser

TABLE I. Layer structure of the (Ga, In)P/GaAs tandem solar cell including nominal layer thickness and fundamental band gap (ind., indirect).

\begin{tabular}{llrc}
\hline \hline & & $\begin{array}{c}\text { Thickness } \\
(\mathrm{nm})\end{array}$ & $E_{g}(\mathrm{eV})$ \\
\hline Top cell & (Al,In)P:Si (window) & 25 & 2.36 (ind.) \\
& (Ga,In)P:Si (emitter) & 140 & 1.84 \\
& (Ga,In)P:Zn (base) & 730 & 1.84 \\
& (Al,Ga,In)P:Zn (BSF) & 70 & 2.13 \\
Tunnel junction & (Al,Ga)As:C & 64 & 2.05 \\
& (Al,Ga)As:C & 28 & 2.00 \\
& GaAs:Te & 15 & 1.39 \\
Bottom cell & (Al,Ga)As:Si & 30 & 2.05 \\
& (Al,Ga)As:Si & 32 & 2.05 \\
& GaAs:Si (emitter) & 80 & 1.42 \\
& GaAs:Zn (base) & 3600 & 1.42 \\
Buffer & (Al,Ga)As:C (BSF) & 165 & 1.95 \\
Substrate & GaAs & 1000 & 1.42 \\
\hline \hline & GaAs (1 0 0) & & 1.42 \\
\hline
\end{tabular}


mechanically chopped at $777 \mathrm{~Hz}$ (hereafter, pump) and a monochromatized beam from a 150-W quartz-halogen-tungsten (QTH) lamp (hereafter, probe). Direct reflectance of the probe beam containing average (dc) and modulated (ac) components is detected with a Si photodiode connected to a preamp and a lock-in amplifier. The PR signal is defined as the spectral ac/dc ratio. In all measurements, 2-nm step sweeps 6 are used for the probe beam. A description of the setup and further references on the technique can be found in Ref. [13].

\section{RESULTS}

\section{A. Photoreflectance}

The calculated absorption profile of the 325-nm pump light under normal incidence across the first layers of the cell structure reveals that about $20 \%$ of the incoming light intensity is transmitted across the (Al,In)P window layer. Further absorption at the (Ga,In)P emitter of the TC reduces the light intensity reaching the top-cell $p$ - $n$ junction to about $0.001 \%$. The following 730 -nm-thick (Ga,In)P-base region virtually ensures that no pump light is transmitted to deeper layers in the structure.

7 Despite full absorption of pump light occurring at the top cell, the PR spectrum shown in the top panel in Fig. 2 reveals that 325 -nm light pump is actually able to modulate the whole device structure, with neat signatures attributed to the top window, $\mathrm{TC}$, and $\mathrm{BC}$ going from high to low energies. Notice that PR is sensitive to direct transitions only, and consequently the signature attributed to the (Al,In)P window at $2.62 \mathrm{eV}$ corresponds to its direct gap. The lower panel in Fig. 2 shows a second PR spectrum obtained under identical conditions but including a variable neutral-density filter (NDF) attenuating the laser pump beam. The pump intensity is in this case lowered down to a level at which the bottom-cell signatures lie below the noise level (mind the reduction in the amplitude of signatures in the top-cell range by about one order of magnitude). These two PR spectra are later associated with the existence and absence of a measurable LC in the device.

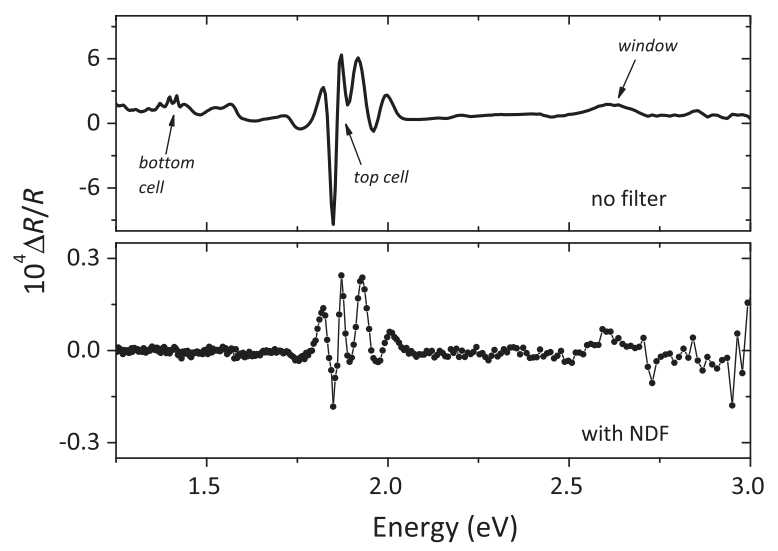

FIG. 2. PR spectra of the $(\mathrm{Ga}, \mathrm{In}) \mathrm{P} / \mathrm{GaAs}$ tandem solar cell using a 325-nm laser line without NDF (upper) and with NDF (lower).

\section{B. Spectral photovoltage}

Figure 3 shows the spectral photovoltage $V_{\mathrm{OC}}$ recorded simultaneously during PR measurements shown in Fig. 2, together with the corresponding $\mathrm{dc}$ reflectance (as recorded, in volts). Three $V_{\mathrm{OC}}$ spectra are shown: (i) using an (unfiltered) pump and probe (dots), with PR revealing a bottom-cell signature; (ii) using a (filtered) pump and probe, such that the PR response from bottom cell lies below noise level (black line); and (iii) a third spectrum using the probe beam only (thus PR silent), with the pump laser turned off (light line).

The band gaps of $\mathrm{TC}$ and $\mathrm{BC}$ (vertical dotted lines) divide the energy spectrum into three regions labeled I, II, and III in Fig. 3. Region I corresponds to the transparency region of the device $(E<1.42 \mathrm{eV})$; region II corresponds to the absorption range of the $\mathrm{BC}$ only $(1.42 \mathrm{eV}<E<1.84 \mathrm{eV})$; and region III also includes the absorption of light at the TC $(E>1.84 \mathrm{eV})$. From Fig. 3, it can be observed that $V_{\mathrm{OC}}$ from the probe beam alone at energies below the $\mathrm{BC}$ band gap at $1.42 \mathrm{eV}$ (region I) is zero, in agreement with no photogeneration occurring within the device. The same applies at the high-energy end of the spectrum in region III due to the decaying spectral content of the QTH lamp. The contribution to $V_{\mathrm{OC}}$ generated by the probe beam is clearly resolved for energies within region II. The absorption range of the $\mathrm{BC}$ is characterized by a fairly constant photovoltage with a superimposed Fabry-Perot oscillation (the anticorrelation $V_{\mathrm{OC}}$ maximum and dc-reflectance minimum confirms the interferometric origin of the rippling). The inflection point from which the photovoltage enters in region III and decays monotonically with increasing energy corresponds to the absorption threshold of the $\mathrm{TC}$ at $1.84 \mathrm{eV}$, in agreement with the corresponding PR signature in Fig. 2. Above this energy, the photovoltage generated by the probe beam

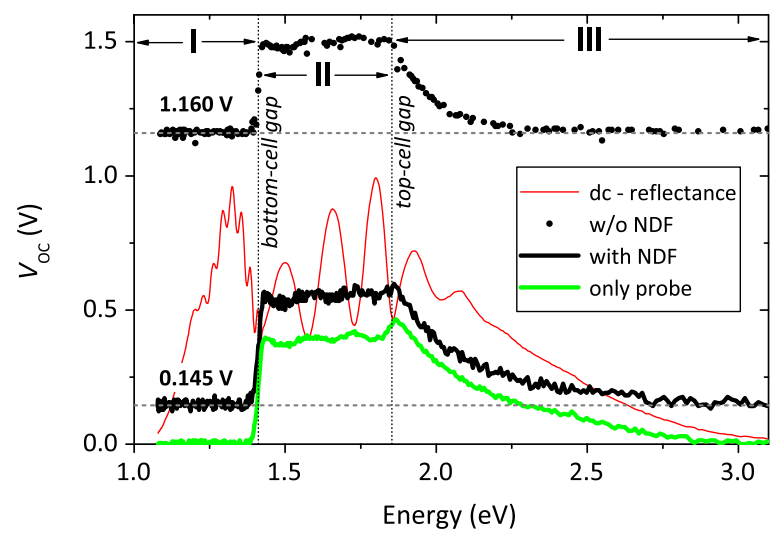

FIG. 3. Photovoltage recorded during PR spectra: under full pump intensity (no NDF, dots); under filtered pump intensity (with NDF, black line); and with the laser off (only probe, green line). de reflectance is also shown (thin red line). Regions I, II, and III correspond to the transparency region, absorption range of $\mathrm{BC}$ alone, and joint absorption at $\mathrm{TC}$ and $\mathrm{BC}$, respectively. 
includes contributions from both top and bottom subcells, the latter resulting from incomplete absorption at TC.

$V_{\mathrm{OC}}$ spectra obtained under the pump and probe with and without NDF appear shifted by 0.145 and $1.160 \mathrm{~V}$, respectively, in comparison to the case of the probe beam alone in the transparency and high-energy regions. Such values correspond to the photovoltage generated by a $325-\mathrm{nm}$ laser line in the device for the two different laser-beam intensities, which according to previous calculations is fully absorbed in the TC in every case. The contribution to $V_{\mathrm{OC}}$ from the pump beam has two components: (i) direct photogeneration at TC, where the beam is fully absorbed, and (ii) eventual coupled photogeneration at BC stemming from TC luminescence (LC). We shall rewrite Eq. (1) for $V_{\mathrm{OC}}$ in region III as

$$
\begin{aligned}
V_{\mathrm{OC}}^{\mathrm{III}, \mathrm{p} \& \mathrm{p}}= & \frac{n^{\mathrm{TC}} k T}{q} \ln \left(\frac{x \times I_{\text {pump }}+I_{\text {probe }}^{\mathrm{TC}}(\lambda)}{I_{0}^{\mathrm{TC}}}\right) \\
& +\frac{n^{\mathrm{BC}} k T}{q} \ln \left(\frac{I_{\text {probe }}^{\mathrm{BC}}(\lambda)+I^{\mathrm{LC}}}{I_{0}^{\mathrm{BC}}}\right) .
\end{aligned}
$$

As explained before, the expression includes one log term per cell with the corresponding prefactor and different current contributions at each subcell from the various active light sources. The wavelength dependency of the probebased photogenerated current is specifically maintained in the expression reflecting the varying spectral content of the QTH lamp, in contrast to the wavelength-independent contribution from the pump. The effect of pump-beam photogeneration at the TC is included in the first, TCrelated log term (expressed as $x \times I_{\text {pump }}$, where $x$ accounts for different pump intensities). The eventual activation of $\mathrm{LC}$ is also included via $I^{\mathrm{LC}}$ in the $\mathrm{BC} \log$ term. Notice the presence of probe-beam photogenerated currents in both $\log$ terms accounting for wavelength-dependent partial transparency of the TC within its absorption range.

A similar expression can also be given for $V_{\mathrm{OC}}$ in transparency region I. For the case of the probe beam alone, the result is trivial with $I_{\text {probe }}=0$. For pump-andprobe spectra,

$V_{\mathrm{OC}}^{\mathrm{I}, \mathrm{p} \& \mathrm{p}}=\frac{n^{\mathrm{TC}} k T}{q} \ln \left(\frac{x \times I_{\mathrm{pump}}+0}{I_{0}^{\mathrm{TC}}}\right)+\frac{n^{\mathrm{BC}} k T}{q} \ln \left(\frac{0+I^{\mathrm{LC}}}{I_{0}^{\mathrm{BC}}}\right)$,

the major difference with respect to the previous expression for region III being the absence of probe-beam photogenerated currents for $E<1.42 \mathrm{eV}$. The latter expression confirms the origin of $V_{\mathrm{OC}}$ in transparency region I stemming from pump-beam photogeneration and allows the distinction of its two contributions: direct photogeneration at TC and related coupled luminescence generating at the BC. It is, in principle, possible to adjust the intensity of the pump beam (reducing the factor $x$ in the previous expression) as to effectively make LC negligible. Should this be the case, $V_{\mathrm{OC}}$ in transparency region I recorded in pump-and-probe spectra would be originated solely at the TC by direct photogeneration of the pump beam [the last term of Eq. (3) drops off]. Indeed, this is the case of the pump-and-probe measurement performed with NDF.

To demonstrate this, Fig. 4 shows the same plot as Fig. 3 with both pump-and-probe spectra shifted down by the respective $V_{\mathrm{OC}}$ readings in the transparency range (labeled as $V_{\mathrm{OC}}^{\mathrm{I}}$ ). All three spectra appear now zeroed in the transparency region. Almost perfect overlapping between spectra corresponding to the cases of pump and probe with NDF and just the probe beam can be observed up to the energy corresponding to the TC band gap at $1.84 \mathrm{eV}$. With $V_{\mathrm{OC}}^{\mathrm{I}}$ zeroed, the device response in this energy range is solely controlled by the $\mathrm{BC}$. According to the observations made for Eq. (3), the magnitude of $V_{\mathrm{OC}}^{\mathrm{I}}$ in the spectrum with NDF in Fig. 3 corresponds solely to the photovoltage generated at the TC by the pump beam, with no additional contribution due to $\mathrm{LC}$ at the BC. The overlapping spectra also rule out eventual LC activation by the probe beam alone across the absorption range of the top cell. It is also recalled that no PR signature from the bottom cell could be detected in this measurement, in agreement with the absence of any measurable LC.

However, an offset of some $67 \mathrm{mV}$ in $V_{\mathrm{OC}}$ is found within the absorption range of the $\mathrm{BC}$ for the case of pumpand-probe measurement at full laser intensity (no filter in Fig. 4). Should $V_{\mathrm{OC}}^{\mathrm{I}}$ correspond to the contribution from the TC alone under the action of the pump beam, the photovoltage spectrum under full laser intensity should overlap the photovoltage developed under the probe beam alone, as in the case with NDF. It is then concluded that the offset detected in the photovoltage spectrum of the pump and probe without NDF (after zeroing $V_{\mathrm{OC}}^{\mathrm{I}}$ ) relative to the probe beam alone corresponds indeed to a contribution to the photovoltage originated at the bottom cell upon the action of the laser beam and, consequently, is not spectrally sensitive. In other words, the last term in Eq. (3) does

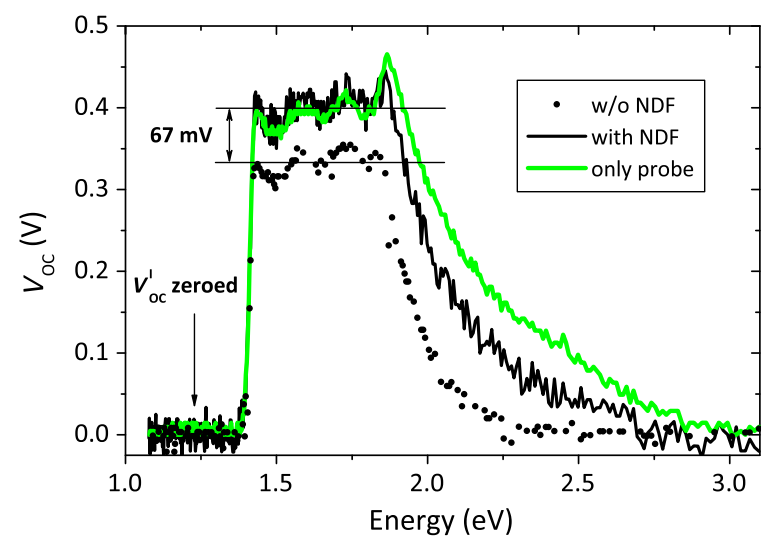

FIG. 4. The same photovoltages from Fig. 3, zeroed to the value in the transparency region, $V_{\mathrm{OC}}^{\mathrm{I}}$. 
not vanish if LC is active. Such an offset, well above the noise level of $V_{\mathrm{OC}}$ spectra, is thus a direct measurement of the $\mathrm{BC}$ contribution to $V_{\mathrm{OC}}$ stemming from LC.

We can estimate the relative luminescence intensity responsible for $\mathrm{BC}$ response under $\mathrm{LC}$ in region II. The analytic expression of the zeroing procedure as applied to the spectrum under pump-and-probe illumination without NDF can be written as

$$
V_{\mathrm{OC}}^{\mathrm{II}, \mathrm{p} \& \mathrm{p}}-V_{\mathrm{OC}}^{\mathrm{I}, \mathrm{p} \& \mathrm{p}}=\frac{n^{\mathrm{BC}} k T}{q} \ln \left(\frac{I_{\mathrm{probe}}^{\mathrm{BC}}(\lambda)}{I^{\mathrm{LC}}}+1\right) .
$$

Notice that $V_{\mathrm{OC}}^{\mathrm{II}, \mathrm{p} \& \mathrm{p}}$ is similar to Eq. (2) with $I_{\text {probe }}^{\mathrm{TC}}(\lambda)=0$, that $V_{\mathrm{OC}}^{\mathrm{I}, \mathrm{p} \& \mathrm{p}}$ is given by Eq. (3), and that the resulting +1 term is not necessarily negligible now. The recorded photovoltage offset of approximately $67 \mathrm{mV}$ corresponds, as indicated in Fig. 4, to the difference between $V_{\mathrm{OC}}^{\mathrm{II}, \text { probe }}$ and Eq. (4), namely,

$$
\begin{aligned}
& V_{\mathrm{OC}}^{\mathrm{II}, \mathrm{probe}}-\left(V_{\mathrm{OC}}^{\mathrm{II}, \mathrm{p} \& \mathrm{p}}-V_{\mathrm{OC}}^{\mathrm{I}, \mathrm{p} \& \mathrm{p}}\right) \\
& =\frac{n^{\mathrm{BC}} k T}{q} \ln \left(\frac{I_{\mathrm{probe}}^{\mathrm{BC}}(\lambda)}{I_{0}^{\mathrm{BC}}}\right)-\frac{n^{\mathrm{BC}} k T}{q} \ln \left(\frac{I_{\mathrm{probe}}^{\mathrm{BC}}(\lambda)}{I^{\mathrm{LC}}}+1\right) .
\end{aligned}
$$

The first term on the right (probe spectrum in region II) is nearly constant except for the interference rippling and equal to $0.4 \mathrm{~V}$. Inverting the expression, we estimate a relative value of $I_{\text {probe }}^{\mathrm{BC}}(\lambda) \approx 4.3 \times 10^{4} \times I^{\mathrm{LC}}$, assuming $26-\mathrm{mV}$ thermal voltage and $n=1.2$ for the BC [14]. Referring back to Sec. II, Eq. (5) corresponds to the magnitude $\Delta V_{\mathrm{OC}}$ of Fig. 1, from which the relative magnitudes of $I_{\text {probe }}^{\mathrm{BC}}(\lambda)$ and $I^{\mathrm{LC}}$ are deduced (to be identified with $I_{1}$ and $I_{2}$ in the figure).

Differences are observed in $V_{\mathrm{OC}}$ spectra in region III, above the top-cell band gap. The nonoverlapping nature of zeroed spectra in region III can be expected from the varying ratios of pump-and-probe photogeneration at the top cell (adding logarithmically to $V_{\mathrm{OC}}$ ), plus the increasing contribution of the bottom cell excited by the probe beam, from the expressions before. Considering the pair of spectra probe alone and filtered pump and probe (setting $x=1$ for convenience), its difference in region III of the zeroed plot in Fig. 4 corresponds to

$$
\begin{aligned}
& V_{\mathrm{OC}}^{\mathrm{III}, \mathrm{NDF}}-V_{\mathrm{OC}}^{\mathrm{III}, \text { probe }}-V_{\mathrm{OC}}^{\mathrm{I}, \mathrm{NDF}} \\
& \quad=\frac{n^{\mathrm{TC}} k T}{q} \ln \left(1+\frac{I_{\text {pump }}^{\mathrm{TC}}}{I_{\text {probe }}^{\mathrm{TC}}(\lambda)}\right)-0.145
\end{aligned}
$$

with $V_{\mathrm{OC}}^{\mathrm{I}, \mathrm{NDF}}=0.145 \mathrm{~V}$. The wavelength dependency of $I_{\text {probe }}(\lambda)$ and thus of the ratio $I_{\text {pump }} / I_{\text {probe }}(\lambda)$ in the log term explains the different curvatures observed in $V_{\mathrm{OC}}$ spectra in region III.

\section{Effect of luminescent coupling on photoreflectance}

Finally, we turn now back to the PR spectrum in Fig. 2 (upper panel) under full pump-beam intensity. With direct evidence of LC operating during the PR measurement, LC is proposed as the modulation mechanism responsible for the bottom-cell PR signature. The LC-mediated modulation mechanism operates right at the same frequency as the chopper modulating the pump beam. The probe beam, in turn, measures changes in reflectance induced by the absorption of light that has been generated at other parts within the structure. We propose that this sort of cascade process, by which light emitted by high-gap materials excites deeper layers of lower band gaps, effectively transmits modulation into the structure far beyond the penetration depth of the pump beam. Furthermore, it is concluded that the absolute distance upon which effective modulation can be transmitted in PR measurements of multilayer semiconductor structures is not necessarily limited by the diffusion length of photogenerated minority carriers $[15,16]$. As an example, optically transmitted modulation by LC satisfactorily describes the propagation of the perturbation across layers expected to block diffusive transport of minority carriers, like the case of tunnel junctions between subcells in open-circuit conditions [17]. The results of Fig. 2 support this conclusion: PR spectra in which LC was selectively activated do show the signature of a buried junction, whereas the same signature becomes PR silent in the absence of LC.

\section{CONCLUSION}

We provide evidence of the impact of LC in spectral photovoltage measurements performed on MJSCs, deriving thereof a simple method to quantify the magnitude of LC as a measurable photovoltage difference. The quantification of LC by means of spectral photovoltage does not require the realization of simultaneous PR measurements; it can rather be performed separately or in combination with alternative characterization measurements like, e.g., QE. Nevertheless, we show that the activation of PR signatures from deep layers as a function of the pump-beam intensity can act as a fair indicator of LC action.

\section{ACKNOWLEDGMENTS}

Financial support from Comunidad de Madrid (MADRID PV, S2013/MAE-2780) is gratefully acknowledged.

[1] M. A. Green, K. Emery, Y. Hishikawa, W. Warta, and E. D. Dunlop, Solar cell efficiency tables v. 47, Prog. Photovoltaics 24, 3 (2016).

[2] M. A. Steiner, J. F. Geisz, T. E. Moriarty, R. M. France, W. E. McMahon, J. M. Olson, S. R. Kurtz, and D. J. Friedman, Measuring I-V curves and subcell photocurrents 
in the presence of luminescent coupling, IEEE J. Photovoltaics 3, 879 (2013).

[3] T. Kirchartz, U. Rau, M. Hermle, A. W. Bett, A. Helbig, and J. H. Werner, Internal voltages in GaInP/GaInAs/Ge multijunction solar cells determined by electroluminescence measurements, Appl. Phys. Lett. 92, 123502 (2008).

[4] D. Friedman, J. Geisz, and M. Steiner, Analysis of multijunction solar cell current-voltage characteristics in the presence of luminescent coupling, IEEE J. Photovoltaics 3, 1429 (2013).

[5] C. Baur, M. Hermle, F. Dimroth, and A. W. Bett, Effects of optical coupling in III-V multilayer systems, Appl. Phys. Lett. 90, 192109 (2007).

[6] S. H. Lim, J. J. Li, E. H. Steenbergen, and Y. H. Zhang, Luminescence coupling effects on multijunction solar cell external quantum efficiency measurement, Prog. Photovoltaics 21, 344 (2013).

[7] M. Z. Shvarts, M. A. Mintairov, V. M. Emelyanov, V. V. Evstropov, and V. M. Lantratov, Method for direct measurements of luminescent coupling efficiency in concentrator MJ-SCs, AIP Conf. Proc. 1556, 147 (2013).

[8] M. A. Steiner and J.F. Geisz, Non-linear luminescent coupling in series-connected multijunction solar cells, Appl. Phys. Lett. 100, 251106 (2012).

[9] H. Mackel and A. Cuevas, The spectral response of the open-circuit voltage: a new characterization tool for solar cells, Sol. Energy Mater. Sol. Cells 81, 225 (2004).

[10] M. Cardona, Modulation Spectroscopy (Academic, New York, 1969).
[11] I. García, I. Rey-Stolle, B. Galiana, and C. Algora, A 32.6\% efficient lattice-matched dual-junction solar cell working at 1000 suns, Appl. Phys. Lett. 94, 053509 (2009).

[12] T. Sharma and T. Hosea, A method of obtaining simultaneous complementary spectroscopic information on selfassembled quantum dots, Jpn. J. Appl. Phys. 48, 082301 (2009).

[13] D. Fuertes Marrón, E. Cánovas, I. Artacho, C. R. Stanley, M. Steer, T. Kaizu, Y. Shoji, N. Ahsan, Y. Okada, E. Barrigón, I. Rey-Stolle, C. Algora, A. Martí, and A. Luque, Application of photoreflectance to advanced multilayer structures for photovoltaics, Mater. Sci. Eng. B 178, 599 (2013).

[14] P. Espinet, I. Rey-Stolle, and C. Algora, Advanced characterization of multijunction solar cells with electroluminescence, in 2009 Spanish Conference on Electron Devices, (IEEE Conference Publications, 2009), p. 390.

[15] M. Motyka, R. Kudrawiec, and J. Misiewicz, On the deepness of contactless electroreflectance probing in semiconductor structures, Phys. Status Solidi (a) 204, 354 (2007).

[16] Y. Mochizuki, T. Ishii, and M. Mizuta, Role of minoritycarrier diffusion in photoreflectance measurements of epitaxial GaAs wafers, Jpn. J. Appl. Phys. 34, 6106 (1995).

[17] E. Cánovas, D. F. Marrón, A. Martí, A. Luque, A. W. Bett, F. Dimroth, and S. P. Philipps, Photoreflectance analysis of a GaInP/GaInAs/Ge multijunction solar cell, Appl. Phys. Lett. 97, 203504 (2010). 